Z. klin. Chem. u. klin. Biochem.

9. Jg., S. 438-442, September 1971

\title{
Über den Zn-Transport bei Ascites-Tumorzellen
}

\author{
Von Th. GüNTHER und F. DORN \\ Zentralinstitut für Biochemie und Biophbysik der Freien Universität Berlin
}

(Eingegangen am 11. Juni 1971)

Die ${ }^{65} \mathrm{Zn}$-Aufnahme und ${ }^{65} \mathrm{Zn}$-Abgabe erfolgen bei Ascites-Tumorzellen unabhängig vom Zellstoffwechsel. Sulfhydrylreagenzien erhöhen die Geschwindigkeit; Inkubation bei $0^{\circ}$ und Änderung des physiologischen $\mathrm{pH}$-Wertes senken die Geschwindigkeit des ${ }^{65} \mathrm{Zn}$-Transportes. Wahrscheinlich sind Proteine der Zellmembran am $\mathrm{Zn}$-Transport beteiligt. Die $\mathrm{Zn}$ =Anreicherung in der Zelle führen wir auf Komplexbildung zurück.

\section{Zn-transport in Ascites tumour cells}

The uptake and release of ${ }^{65} \mathrm{Zn}$ by Ascites tumour cells is independent of cell metabolism. Sulphydryl reagents increase the rate of uptake and release of ${ }^{65} \mathrm{Zn}$. The rate of ${ }^{65} \mathrm{Zn}$ transport is decreased by incubation at $0^{\circ}$ and by changes in the physiological pH. Proteins of the cell membrane are probably involved in $\mathrm{Zn}$ transport. The accumulation of $\mathrm{Zn}$ is attributed to complex formation within the cell.

$\mathrm{Zn}$ ist ein lebensnotwendiges Bioelement und Bestandteil zahlreicher Enzyme. Bei seinem Mangel treten mannigfaltige Störungen auf. Zn kommt vorzugsweise intrazellulär vor. Vom Zn-Bestand eines Organs'befinden sich etwa $99 \%$ in den Zellen. Zellen verschiedener Organe unterscheiden sich in ihrem $\mathrm{Zn}-\mathrm{Gehalt}$ und ${ }^{65} \mathrm{Zn}$-turnover.

Leber- und Nierenzellen haben einen hohen, Muskelzellen einen niedrigen intrazellulären $\mathrm{Zn}$-Gehalt. Leberund Nierenzellen nehmen ${ }^{65} \mathrm{Zn}$ mit hoher, Muskelzellen nur mit geringer Geschwindigkeit auf (1). In der vorliegenden Arbeit soll untersucht werden, wie $Z_{n}$ durch die Zellmembran in Zellen und aus Zellen transportiert wird und wie die intrazelluläre Anreicherung von $\mathrm{Zn}$ erklärt werden kann.

\section{Methodik}

Für die Versuche verwendeten wir Ehrlich-Mäuseascites-Tumorzellen 9 bis 12 Tage nach intraperitonealer Inokulation.

Zur Messung der ${ }^{65} \mathrm{Zn}$-Aufnahme wurden die Zellen in einer Salzlösung, die $135 \mathrm{~mm} \mathrm{NaCl}, 5,4 \mathrm{mM} \mathrm{KCl}, 13,5 \mathrm{~mm} \mathrm{NaHCO}$ und $5,5 \mathrm{~ms}$ Glucose enthielt, mit $0,1 \mu \mathrm{C} / \mathrm{ml}{ }^{65} \mathrm{Zn}$ inkubiert [Radiochemical Centre Amersham, Spezifische Aktivität: $5 \mathrm{mC}$ / $2,71 \mathrm{mg} \mathrm{Zn}$ ]. Die $\mathrm{Zn}_{\mathrm{n}}$ Konzentration dieser Lösung war $0,4 \mu \mathrm{M}$, ihr $\mathrm{pH}$ betrug 7,3. Die Zellkonzentration wurde, um eine gute Reproduzierbarkeit der Versuche zu erreichen, immer auf $5 \%$ $[\nabla / v]$ eingestellt, denn bei geringerer Zellkonzentration exgibt sich eine relativ höhere ${ }^{65} \mathrm{Zn}$-Aufnahme. Nach Beendigung der Inkubation, die bei $37^{\circ}$ unter Schütteln erfolgte, wurden die Zellen bei $4000 \mathrm{~g}$ abzentrifugiert und einmal in der gleichen auf $0^{\circ}$ gekühlten Lösung ohne bzw. mit Zusatz von $0,1 \mathrm{~mm} \mathrm{ZnCl}_{2}$ gewaschen. Die ${ }^{65} \mathrm{Zn}$-Aktivität im Überstand nach dem Waschen ohne bzw. mit Zusatz von $\mathrm{ZnCl}_{2}$ war gering, so daß die extrazelluläre ${ }^{65} \mathrm{Zn}$-Aktivität $z$ wischen den sedimentierten Zellen $0,2-0,5 \%$ der zellulären Aktivität betrug und zu vernachlässigen ist.

Zur Messung des $\mathrm{Zn}$-Efflux wurden die Zellen in gleicher Konzentration durch $20 \mathrm{Min}$. Inkubation bei $37^{\circ} \mathrm{mit} 0,5 \mu \mathrm{C}{ }^{65} \mathrm{Zn} / \mathrm{ml}$ beladen, zweimal mit der oben beschriebenen gekühlten Salzlösung, die auf vier Teile einen Teil inaktiviertes Ascitesserum enthielt, gewaschen und in inaktiviertem Ascitesserum, das im Verhältnis 1:1 mit der Salzlösung verdünnt war, unter Zusatz von
5,5 mM Glucose inkubiert. $\mathrm{Zu}$ verschiedenen Zeiten wurden aliquote Teile entnommen und bei $1000 \mathrm{~g}$ zentrifugiert. In den Zellsedimenten wurde die ${ }^{65} \mathrm{Zn}$-Aktivität nach Auflösen der Zellen in $1 \mathrm{ml} 1 \mathrm{~N} \mathrm{NaOH}$ unter $20 \mathrm{Min}$. Erwärmen auf $60^{\circ}$ im Bohrlochkristall (Fa. Frieseke und Hoepfner) gemessen.

Der Proteingehalt wurde nach Lowry und Mitarbeiter (2) bestimmt. Der $\mathrm{Zn}_{\mathbf{n}}$-Gehalt der Zellen wurde nach Veraschen in konz. $\mathrm{HNO}_{3}$, der $\mathrm{Zn}$-Gehalt des Ascitesserums nach Verdünnen im Atomabsorptionsspektrometer gemessen (1).

\section{Ergebnisse}

Mäuseascites-Tumorzellen haben einen intrazellulären Zn-Gehalt von 1,50 mMol/kg Trockensubstanz. Der Zn-Gehalt im Ascitesserum beträgt $17 \mu \mathrm{Mol} / \mathrm{l}$.

Die Geschwindigkeit der ${ }^{65} \mathrm{Zn}$-Aufnahme ist in Abbildung 1 dargestellt. Zn wird mit hoher Geschwindigkeit in Ascites-Tumorzellen aufgenommen. Die Geschwindigkeit der $\mathrm{Zn}$-Aufnahme steigt mit der extrazellulären ${ }^{65} \mathrm{Zn}$-Konzentration (Abb. 2) linear an. Waschen mit einer auf $0^{\circ}$ gekühlten, inaktives $Z$ n enthaltenden Lösung setzt die ${ }^{65} \mathrm{Zn}$-Aktivität der Zellen nur wenig therab (Abb. 2). Eine Sättigungskinetik der ${ }^{65} \mathrm{Zn}$-Aufnahme war im Gegensatz zu Hela-Zellen (3) bis zu einer extrazellulären Konzentration von $0,3 \mathrm{~mm}$ nicht festzustellen.

Das pH-Optimum der Zn-Aufnahme liegt bei $\mathrm{pH} 7,3$ (Abb. 3). Mit steigender Temperatur durchläuft die Aufnahmegeschwindigkeit ein Optimum bei $40^{\circ}$ (Abb. 4).

$\mathrm{Da}$ der Zn-Bestand eines Organs bzw. Gewebes sich zum größten Teil in den Zellen befindet (s. o.) (1) und Zn mit Proteinen, Aminosäuren usw. sehr stabile Chelate bildet, ist die $\mathrm{Zn}$-Ionenaktivität in der extra- und intrazellulärẹn Flüssigkeit wahrscheinlich sehr niedrig und nicht bekannt. Demzufolge ist eine Aussage über den Zn-Transport in bezug auf die Richtung des elektrochemischen Potentials nicht möglich, d. h. aus der Verteilung des $\mathrm{Zn}$ läßt sich unter Berücksichtigung des Membranpotentials nicht entscheiden, ob es einen (primären oder sekundären) aktiven Transport von $\mathrm{Zn}$ gibt. $\mathrm{Da}$ aktiver Substanztransport von energieliefernden 


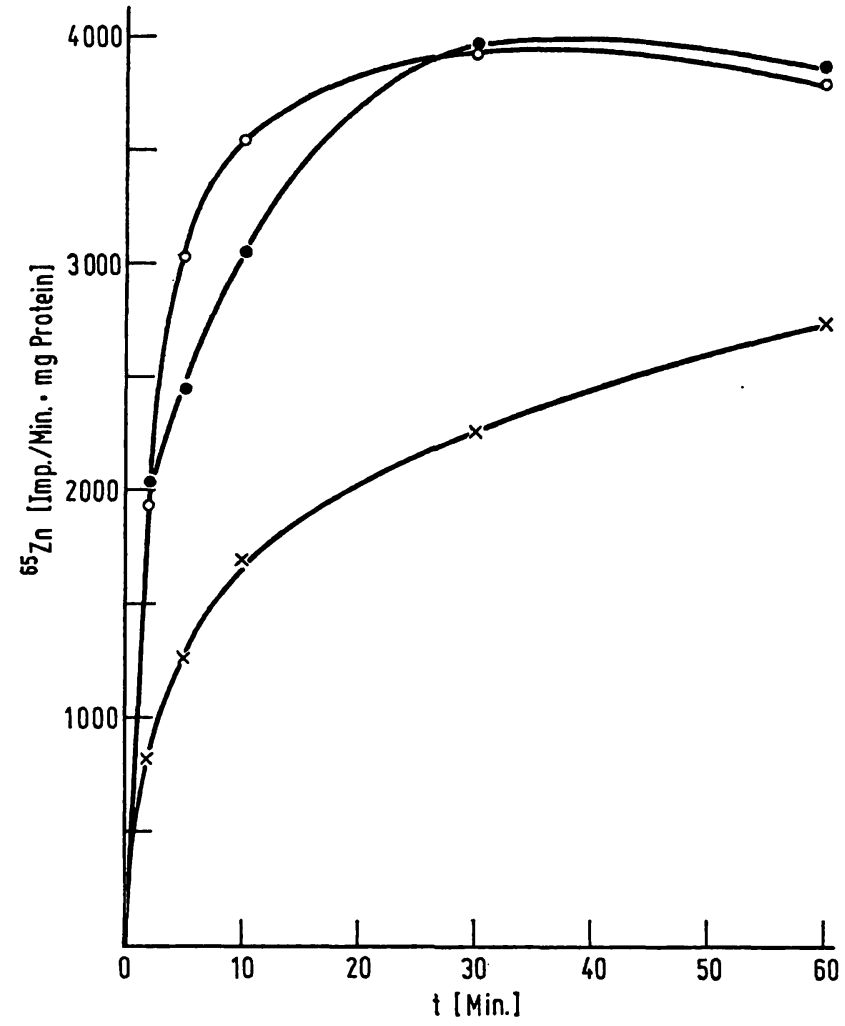

Abb. 1

sZn-Aufnahme als Funktion der Inkubationszeit

Die Zellen wurden $10 \mathrm{Min}$. bei $37^{\circ}$ vorinkubiert, Start durch Zugabe von ${ }^{\circ} \mathrm{Zn}$. Nach verschiedenen Zeiten wurden die Zellen abzentrifugiert und einmal in gekühltem Inkubationsmedium gewaschen. $2,9 \cdot 10^{4} \mathrm{Imp}$./Min. entsprechen $1 \mathrm{nMol} \mathrm{Zn}$. Die extrazelluläre $\mathrm{Zn}$ Konzentration betrug $0,4 \mu \mathrm{M} . \propto \circ=1 \mathrm{~mm} N$-Ảthylmaleinimid; $\bullet-0,1 \mathrm{~mm} \mathrm{HgCl} ; \times-x=$ Kontrolle

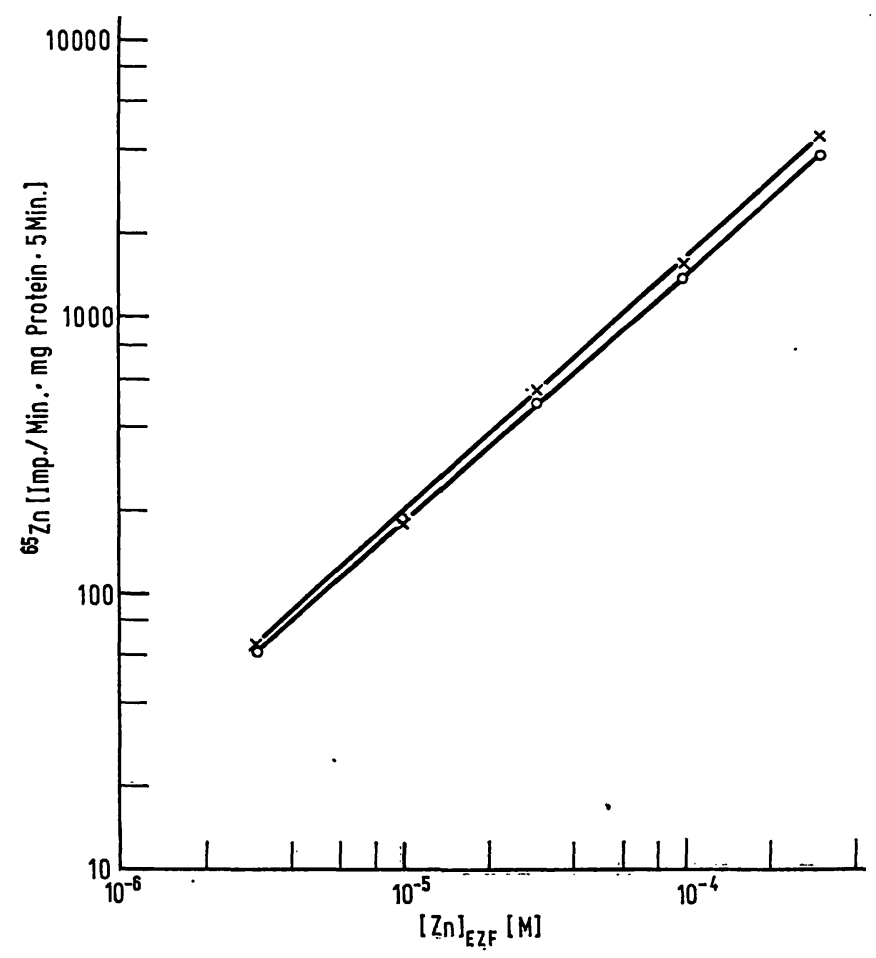

Abb. 2

Abhängigkeit der ${ }^{\circ} \mathrm{Zn}$-Aufnahme von der extrazellulären $\mathrm{Zn}$-Konzentration. Die Zellen wurden 10 Min. vorinkubiert. Start durch Zugabe von ${ }^{\circ} \mathrm{Zn}$. Nach 5 Min. wurden die Zellen abzentrifugiert und einmal in gekühitem Inkubationsmedium ohne $[x-x]$ und mit $\mathrm{Zn}-\mathrm{Zusatz}$ $[0 \rightarrow$ gewaschen

$1,51 \cdot 10^{4} \mathrm{Imp}$./Min. entsprechen in diesen Versuchen wegen der geringer gewähiten spezifischen Aktivität $100 \mathrm{nMol} \mathrm{Zn}$

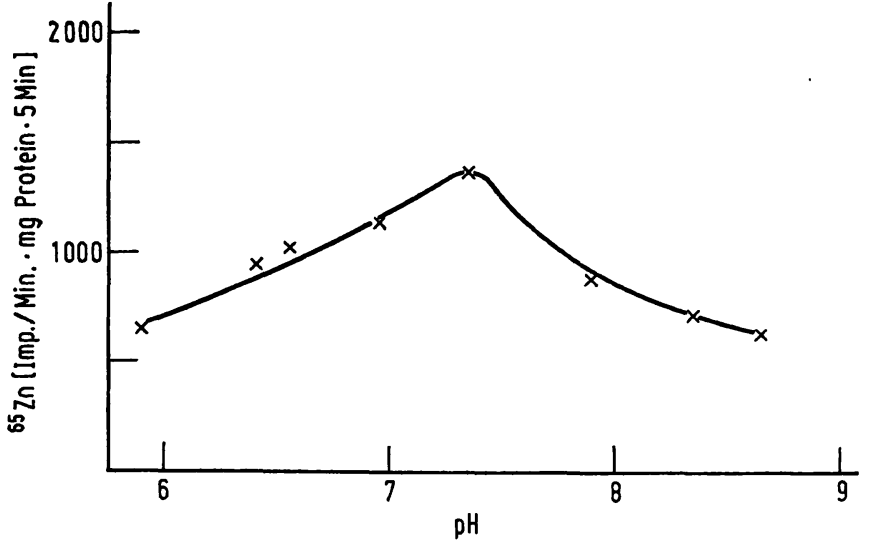

Abb. 3

Abhängigkeit der ${ }^{\circ} \mathrm{Zn}$-Aufnahme vom $\mathrm{pH}$

Die Zellen wurden bei $37^{\circ} 10 \mathrm{Min}$. in Inkubationsmedium vorinkubiert das $10 \mathrm{~mm}$ Puffer enthielt, und zwar:

$\begin{array}{ll}\text { unter pH 6,0 } & \text { Acetatpuffer } \\ \text { zwischen pH 6,5 und 7,0 } & \text { Imidazol/Salzsäure } \\ \text { und über pH 7,5 } & \text { Tris/Salzsäure }\end{array}$

und über pH 7,5 Tris/Salzsäure Start durch Zugabe von ${ }^{\circ} \mathrm{BZn}$. Nach $5 \mathrm{Min}$. wurden die Zellen ab-
zentrifugiert und einmal in gekühltem, gepuffertem Inkubationsmedium gewaschen

$2,9 \cdot 10^{4}$ Imp./Min. entsprechen $1 \mathrm{nMol} \mathrm{Zn}$. Die extrazelluläre $\mathrm{Zn}$ Konzentration betrug $0.4 \mu \mathrm{M}$

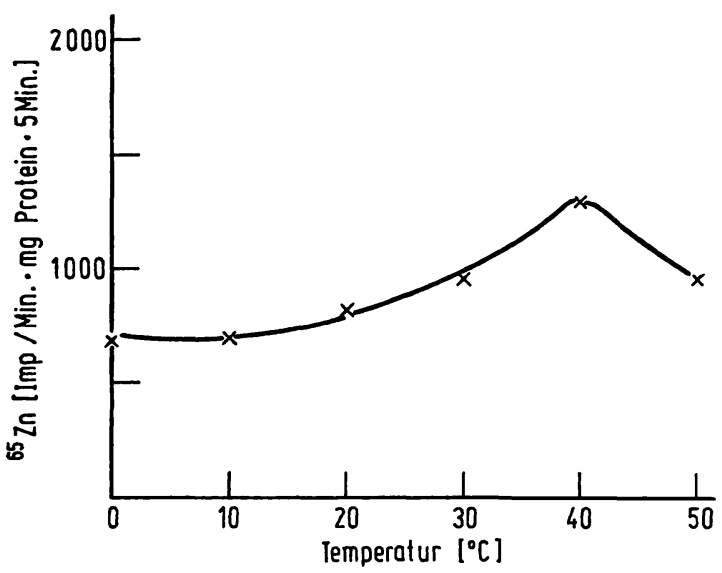

Abb. 4

Abhängigkeit der ${ }^{8} \mathrm{Zn}$-Aufnahme von der Temperatur Die Zellen wurden $10 \mathrm{Min}$. bei der entsprechenden Temperatur vorinkubiert. Start durch Zugabe von ${ }^{\circ} \mathrm{Zn}$, Inkubationszeit $5 \mathrm{Min}$. Die Zellen wurden in gekühltem Inkubationsmedium einmal gewaschen. 2,9 $\cdot 10^{\prime}$ Imp./Min. entsprechen $1 \mathrm{nMol} \mathrm{Zn}$. Die extrazelluläre $\mathrm{Zn}$ Konzentration betrug $0,4 \mu \mathrm{M}$

Stoffwechselreaktionen abhängig ist, prüften wir den Einfluß verschiedener Inhibitoren auf die $\mathrm{Zn}$-Aufnahme.

Tabelle 1 zeigt, daß Stoffwechselgifte wie 2,4-Dinitrophenol, Azid, Cyanid, Fluorid und Jodacetat oder Inkubation ohne Glucose keinen wesentlichen Einfluß haben. Ähnliche Ergebnisse wurden an Pflanzenzellen und Fischeiern gefunden $(4,5,6)$. Die $\mathrm{Zn}$-Aufnahme ist also nicht abhängig vom Zellstoffwechsel. Strophanthin, das die membrangebundene $\mathrm{Na}$ - und $\mathrm{K}$-aktivierbare ATPase und den aktiven Na-K-Transport spezifisch hemmt, hatte keinen signifikanten Einfluß auf die Zn-Aufnahme. Damit übereinstimmend hatte die Erniedrigung der extrazellulären Na-Konzentration (isoosmotischer Ersatz durch Cholinchlorid) und Erhöhung der extrazellulären K-Konzentration ebenfalls keine Wirkung. Daraus folgt, $\mathrm{da}$ die $\mathrm{Zn}$-Aufnahme nicht mit dem Na-K-Transport 
Tab. 1

Einfluß verschiedener Substanzen auf die ${ }^{\circ} \mathrm{Zn}$-Aufnahme in AscitesTumorzellen

Die Zellen wurden mit den verschiedenen Substanzen $10 \mathrm{Min}$. bei $37^{\circ}$ im Wasserbad geschüttelt, danach $5 \mathrm{Min}$. mit ${ }^{\circ} \mathrm{Zn}$ inkubiert, abzentrifugiert und einmal in gekühltem Inkubationsmedium ohne $\mathrm{Zn}$-Zusatz gewaschen. $2,9 \cdot 10^{4}$ Imp./Min. entsprechen $1 \mathrm{nM}$
$\mathrm{n}$

\begin{tabular}{|c|c|c|}
\hline Substanzen & $\begin{array}{c}\text { Konzentration } \\
(\mathrm{mm})\end{array}$ & $\begin{array}{l}\text { Imp./Min. } \cdot \text { mg } \\
\text { Protein } \cdot 5 \text { Min. }\end{array}$ \\
\hline $\begin{array}{l}\text { Kontrolle } \\
\text { ohne Glucose } \\
2,4-\mathrm{D} \text { initrophenol } \\
\mathrm{KCN} \\
\mathrm{NaF} \\
\mathrm{KCN}+\mathrm{NaF} \\
\mathrm{NaN}_{3} \\
\text { Jod-Acetat } \\
\text { Glykokoll } \\
\text { Asparaginsäure } \\
\mathrm{Methionin} \\
\mathrm{Na}-\mathrm{Citrat} \\
\mathrm{Na} \mathrm{a}_{2} \mathrm{HPO} \\
\mathrm{Mg} \\
\mathrm{Ca} \\
\mathrm{Mn} \\
\mathrm{Co} \\
\mathrm{Ni} \\
\mathrm{Cu} \\
\mathrm{Cd} \\
\mathrm{Hg} \\
\mathrm{N}-\mathrm{A} \text { thylmaleinimid } \\
\mathrm{Cystein} \\
\text { EDTA }\end{array}$ & $\begin{array}{r}1 \\
1 \\
10 \\
10 \\
10 \\
1 \\
1 \\
1 \\
1 \\
1 \\
1 \\
1 \\
1 \\
1 \\
3 \\
0,1 \\
0,1 \\
0,1 \\
0,1 \\
0,1 \\
0,1 \\
1 \\
1 \\
1\end{array}$ & $\begin{array}{r}1330 \\
1330 \\
1310 \\
1310 \\
1290 \\
1290 \\
1340 \\
1380 \\
1320 \\
1460 \\
1470 \\
1170 \\
1140 \\
1630 \\
1680 \\
1680 \\
1470 \\
1340 \\
2990 \\
3290 \\
3510 \\
3160 \\
680 \\
40\end{array}$ \\
\hline
\end{tabular}

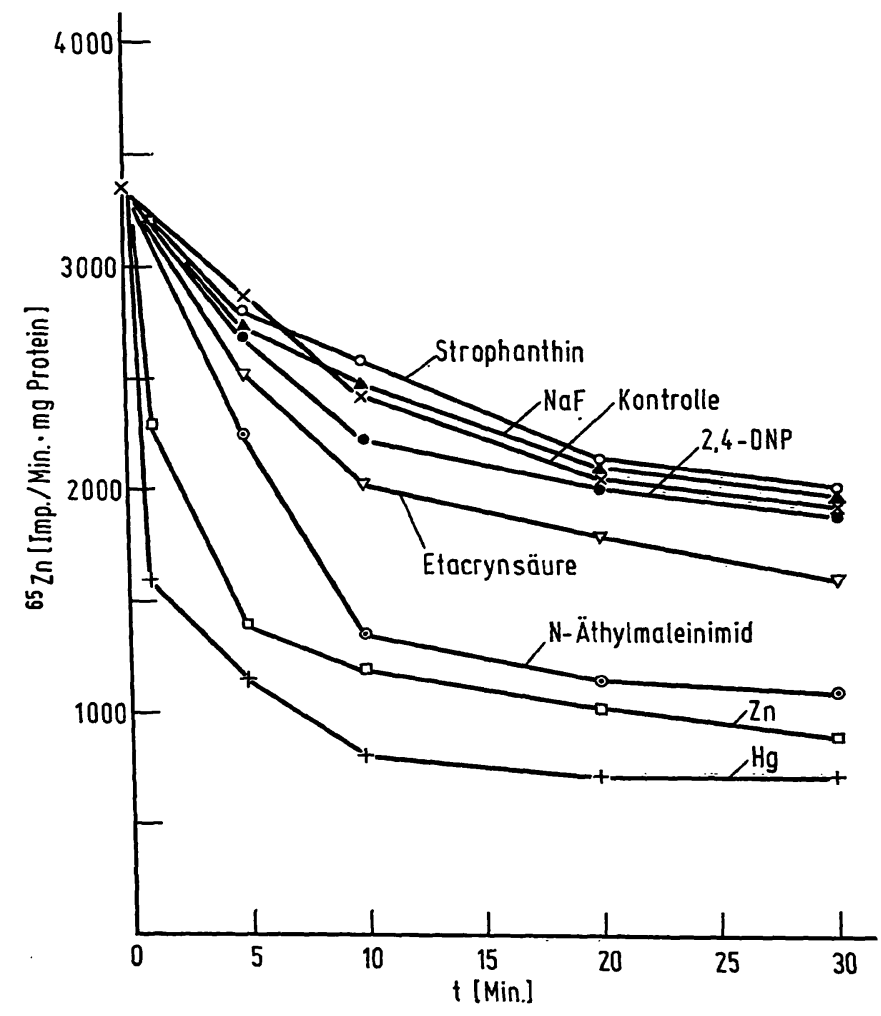

Abb. 5

Einfluß verschiedener Inhibitoren auf den ${ }^{\circ} \mathrm{Zn}$-Efflux Die Zellen wurden $20 \mathrm{Min}$. bei $37^{\circ}$ im Inkubationsmedium mit ${ }^{\circ} \mathrm{Zn}$ vorbeladen, dann in Inkubationsmedium-Ascitesserum $[4: 1]$ zweima gewaschen und in Inkubationsmedium-Ascitesserum [1:1] mit den nhibitoren reinkubiert

$$
\begin{aligned}
& \quad \text { Inhibitor } \\
& \mathrm{Zn}=\left[\mathrm{ZnCl}_{2}\right] \\
& \text { Strophanthin } \\
& \text { NaF } \\
& \text { 2,4-Dinitrophenol } \\
& \text { Etacrynsäure } \\
& \text { N- }- \text { Ăthylmaleinimid } \\
& \mathrm{HgCl}_{2}
\end{aligned}
$$

Konzentration [mM]

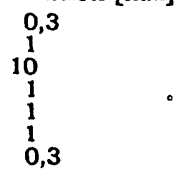

gekoppelt ist. Inkubation in Medien verschiedener Osmolarität, um die Membranpermeabilität infolge einer Zellschwellung zu variieren, führte zu keiner Änderung des ${ }^{65} \mathrm{Zn}$-Transportes.

Dagegen verminderten $\mathrm{Zn}$-bindende Substanzen wie EDTA oder Cystein im Inkubationsmedium die $\mathrm{Zn}$ Aufnahme. Substanzen, die mit Sulfhydrylgruppen reagieren, besonders $\mathrm{Hg}^{++}$und $\mathrm{N}$-Äthylmaleinimid, bewirken eine starke Zunahme der ${ }^{65} \mathrm{Zn}$-Aufnahme (Tab.1, Abb. 1) sowie eine schnellere Einstellung der steadystate-Verteilung.

Die $\mathrm{Zn}$-Aufnahme kann durch andere $z$ weiwertige $\mathrm{Me}$ tallionen wie $\mathrm{Ca}, \mathrm{Mg}, \mathrm{Mn}$, Co und Ni nicht gehemmt werden (Tab. 1). Eine Konkurrenz um gemeinsame Akzeptoren ist also nicht nachzuweisen. Die Beschleunigung der $\mathrm{Zn}$-Aufnahme durch $\mathrm{Cd}$ und $\mathrm{Cu}$ könnte durch Reaktion dieser Ionen mit Membranbestandteilen ähnlich wie durch $\mathrm{Hg}$ zustande kommen.

Das aufgenommene ${ }^{65} \mathrm{Zn}$ kann teilweise wieder aus den Zellen abgegeben werden, wahrscheinlich im Austausch gegen $\mathrm{Zn}$ des Inkubationsmediums, denn bei einer extrazellulären $\mathrm{Zn}$-Konzentration von $0,3 \mathrm{~mm}$ gelangt wesentlich mehr $\mathrm{Zn}$ nach außen als bei den Kontrollansätzen in verdünntem Ascitesserum mit $9 \mu \mathrm{M} \mathrm{Zn}$. Der ${ }^{65} \mathrm{Zn}$-Efflux ist analog zum ${ }^{65} \mathrm{Zn}$-Influx durch Stoffwechselinhibitoren nicht signifikant zu beeinflussen. Dagegen erhöhen $\mathrm{Hg}$ und $\mathrm{N}$-Äthylmaleinimid auch den ${ }^{65} \mathrm{Zn}$-Efflux (Abb.5). Der intrazelluläre ${ }^{65} \mathrm{Zn}$-Gehalt (Abb. 5 und 6); dessen

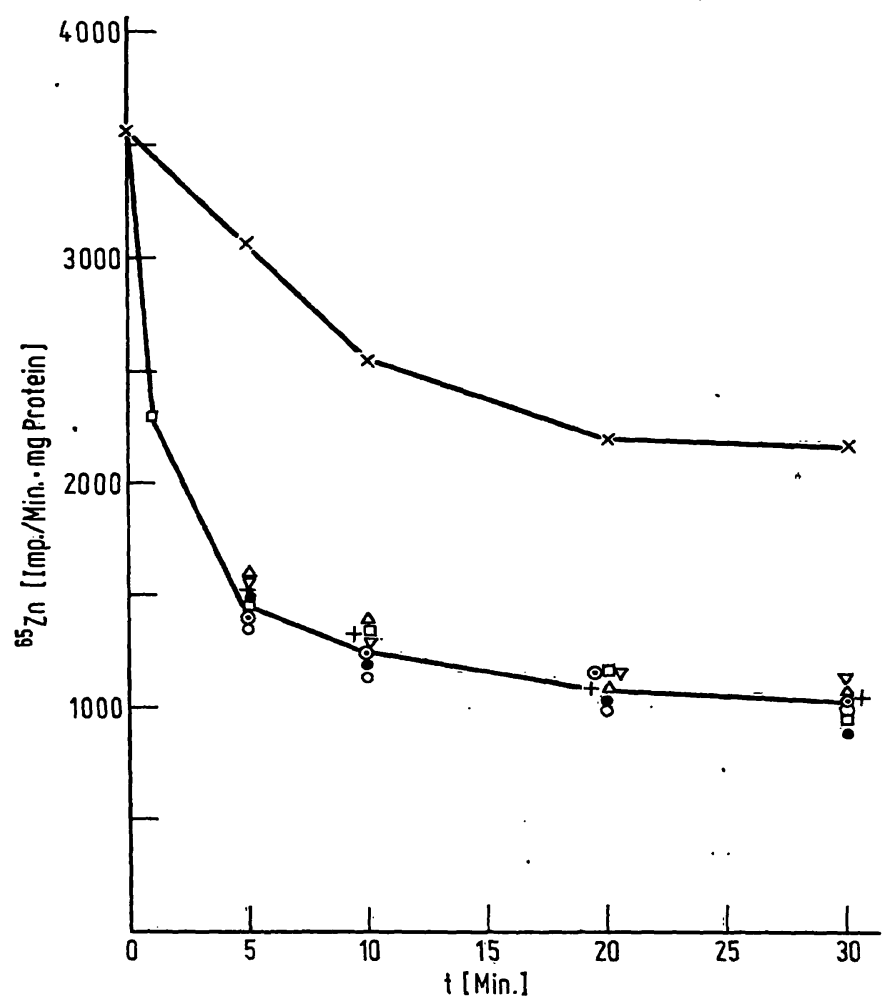

Abb. 6

Einfluß verschiedener Inhibitoren auf den ${ }^{a Z Z n-E f f l u x}$ bei einer

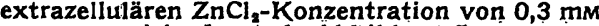
siehe Legende Abbildung 5

$x=$ Kontrolle ohne $\mathrm{ZnCl}_{2} \quad+=\mathrm{KCN}+\mathrm{NaF}$

$\nabla=$ Etacrynsäure $\quad \circ=$ Strophanthin

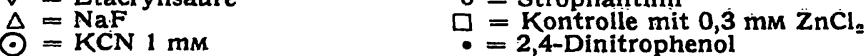


Abnahme den $\mathrm{Zn}$-Efflux darstellt, kann nicht.wie in den übrigen Abbildungen und in der Tabelle in $\mathrm{nMol} \mathrm{Zn}$ umgerechnet werden, denn die spezifische Aktivität des intrazellulären ${ }^{65} \mathrm{Zn}$ kann nicht angegeben werden, weil wir zur Vermeidung einer Zellschädigung infolge zu langer Versuchsdauer die Einstellung des Verteilungsgleichgewichts des. ${ }^{65} \mathrm{Zn}$ nicht abgewartet haben und das intrazelluläre ${ }^{65} \mathrm{Zn}$ in der Zelle wahrscheinlich nicht gleichmäßig verteilt ist.

Als weiteren Test, ob am Zn-Efflux stoffwechselabhängige Reaktionen beteiligt sind, prüften wir den Einfluß von Inhibitoren auf den bei hohen extrazellulären $\mathrm{Zn}$ Konzentrationen verstärkten ${ }^{65} \mathrm{Zn}$-Efflux (Abb. 6). Dabei ergab sich, daß die Stoffwechselinhibitoren auch den erhöhten Zn-Efflux nicht veränderten. Dagegen bewirkte die Inkubation bei $0^{\circ}$ eine starke Abnahme des ${ }^{65} \mathrm{Zn}$ Effluxes sowohl bei einer extrazellulären Zn-Konzentration von $9 \mu \mathrm{M}$ wie auch bei $0,3 \mathrm{~mm} \mathrm{Zn}$ (Abb. 7).

Außerdem untersuchten wir die Wirkung einer gehemmten Proteinbiosynthese und fanden, daß intraperitoneale Injektion von $0,5 \mathrm{mg}$ Cycloheximid pro Maus 18 Stunden vor der Zellentnahme keinen signifikanten Effekt auf ${ }^{65} \mathrm{Zn}$-Aufnahme und ${ }^{65} \mathrm{Zn}$-Abgabe der vorbehandelten Zellen hatte.

\section{Diskussion}

Die ${ }^{65} \mathrm{Zn}$-Aufnahme kann nur zu einem geringen Teil auf ${ }^{65} \mathrm{Zn}$-Bindung an die Zellmembran beruhen, denn beim Waschen mit gekühlter, inaktives $\mathrm{Zn}$ enthaltender Lö-

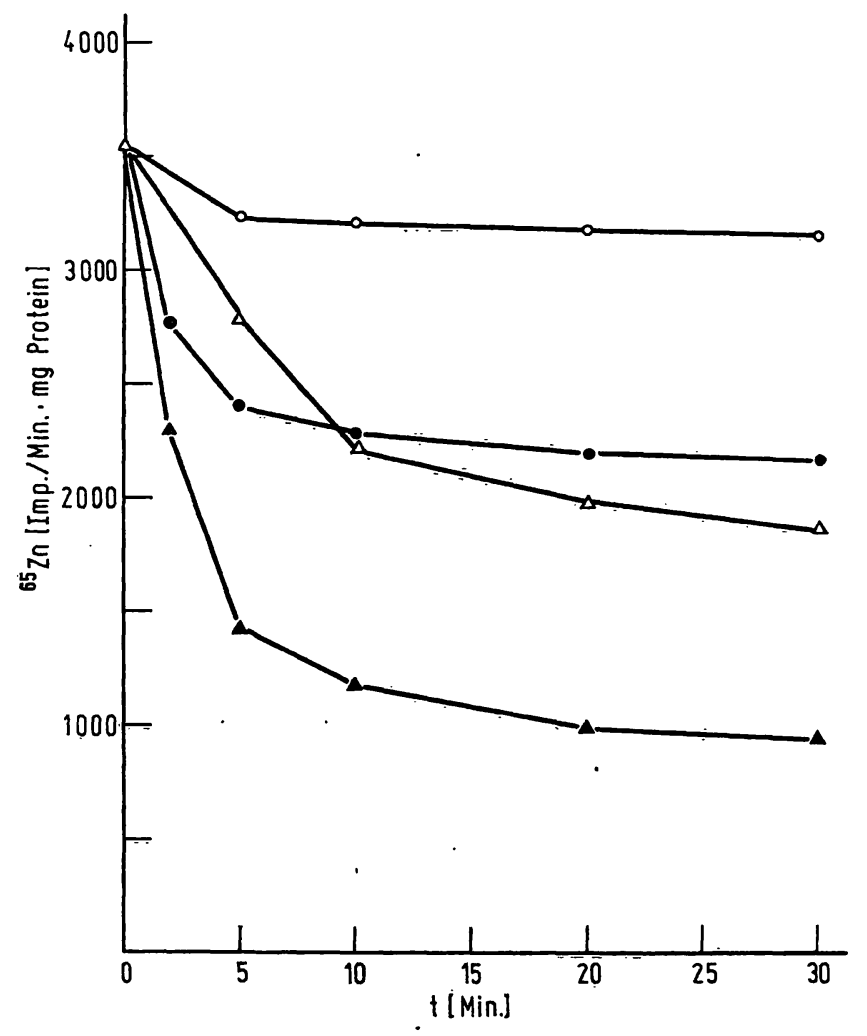

Abb. 7

"Zn-Efflux bei $37^{\circ}[\Delta, \Delta]$ und $0^{\circ}[0, \cdot]$ sowie bei einer extrazellulären $\mathrm{Zn}-\mathrm{K}$ onzentration von $9 \mu \mathrm{M}[\Delta$, , ] bzw. $0,3 \mathrm{mM}[\Delta, \cdot]$ sung wird von den mit Inhibitoren behandelten und den unbehandelten Zellen nur wenig ${ }^{65} \mathrm{Zn}$ entfernt. Das von den Zellen aufgenommene $\mathrm{Zn}$ muß also zum überwiegenden Teil in einer bei $0^{\circ}$ nur geringfügig austauschbaren Fraktion vorliegen. Wahrscheinlich handelt es sich dabei um intrazelluläres $\mathrm{Zn}$. Wie die Inkubation bei $0^{\circ}$ zeigt (Abb. 7), wird hierbei der bei $37^{\circ}$ schnelle $\mathrm{Zn}$ Efflux stark reduziert. Beim Waschen der Zellen mit auf $0^{\circ}$ gekühlten $\mathrm{Zn}$-freien und $\mathrm{Zn}$-haltigen Lösungen wird also hauptsächlich das extrazelluläre und nur wenig intrazelluläres ${ }^{65} \mathrm{Zn}$ entfernt. Das erklärt, weshalb beim Waschen mit $\mathrm{Zn}$-haltiger Lösung gegenüber $\mathrm{Zn}$-freier Lösung nur wenig ${ }^{65} \mathrm{Zn}$ von den Zellen entfernt wird.

Die mit steigender extrazellulärer Zn-Konzentration linear zunehmende ${ }^{65} \mathrm{Zn}$-Aufnahmegeschwindigkeit, die auch bei Pflanzenzellen gefunden wurde (5), eine fehlende Hemmung durch andere zweiwertige Kationen und der fehlende Einfluß von Stoffwechselinhibitoren lassen den Schluß zu, daß bei der Zn-Aufnahme in Ascites-Tumorzellen kein stoffwechselabhängiger aktiver Transport vorliegt. Der $\mathrm{Zn}$-Transport könnte demnach durch Diffusion bzw. Austauschdiffusion erfolgen.

Der Zn-Transport scheint teilweise reversibel zu sein, denn die ${ }^{65} \mathrm{Zn}$-Abgabe aus vorbeladenen Ascites-Tumorzellen verhält sich unter Einwirkung von Stoffwechselinhibitoren und Sulfhydrylreagenzien analog wie die ${ }^{65} \mathrm{Zn}$-Aufnahme. In der Kälte ist die $\mathrm{Zn}$-Abgabe jedoch stärker herabgesetzt als die Zn-Aufnahme. Das mag z. T. daran liegen, daß bei der Messung der ${ }^{65} \mathrm{Zn}$ Aufnahme nicht exakt $z$ wischen ${ }^{65} \mathrm{Zn}$-Bindung an die Zellmembran und intrazellulärer ${ }^{65} \mathrm{Zn}$-Aufnahme $\mathrm{zu}$ differenzieren ist.

Die Zn-Aufnahme in Hela-Zellen zeigte eine Sättigungskinetik. Die maximale Aufnahmegeschwindigkeit wurde bei einer extrazellulären $\mathrm{Zn}$-Konzentration von etwa $0,1 \mathrm{~mm}$ erreicht (3). Man kann daraus auf die Existenz eines geschwindigkeitsbestimmenden Schrittes in der Membran dieser Zellen schließen.

Der $\mathrm{Zn}$-Transport in und aus Ascites-Tumorzellen erfolgt nicht mit seiner maximalen Geschwindigkeit, denn er wird durch Sulfhydrylgruppenblocker wie $\mathrm{Hg}^{2+}$ und N-Äthylmaleinimid stark erhöht. Offenbar können Membranproteine, die mit SH-Reagenzien reagieren, den Transport von $\mathrm{Zn}$ durch die Membran von AscitesTumorzellen verzögern. Wahrscheinlich sind diese Proteine, wie man aus der Wirkung der schlecht permeierenden Schwermetallionen folgern kann, auf der Zellmembran und nicht im Zellinnern lokalisiert. Da sich der ZnTransport mit dem extrazellulären $\mathrm{pH}$ und der Temperatur ändert und vom Stoffwechsél unabhängig ist, kann man den Schluß ziehen, daß der Zn-Transport vom Ladungszustand funktioneller Gruppen und von der Konformation von Proteinen bzw. Lipoproteinen der Zellmembran beeinflußt wird. Vielleicht besteht hierin eine Möglichkeit zur Regulation, denn die Geschwindigkeit des $\mathrm{Zn}$-Transportes in verschiedene Zellen weist erhebliche Unterschiede auf. 
Mit ihrem hohen $\mathrm{Zn}$-Gehalt und ihrem schnellen ${ }^{65} \mathrm{Zn}$ turnover verhalten sich Ascites-Tumorzellen wie Leberund Nierenzellen. Die Ergebnisse an Ascites-Tumorzellen lassen sich daher wahrscheinlich auf diese Zellen übertragen. Muskel- oder Hela-Zellen verhalten sich dagegen anders. Diese Zellen nehmen ${ }^{65} \mathrm{Zn}$ mit wesentlich geringerer Geschwindigkeit auf. Nach Adrenalektomie ist die ${ }^{65} \mathrm{Zn}$-Aufnahme in Muskelzellen im Gegensatz zu Leber und Niere (1) verlangsamt. Glucocorticoide erhöhen im Gegensatz zu Leber und Niere den Zn-Gehalt von Muskelzellen (1) und die ${ }^{65} \mathrm{Zn}$-Aufnahme in HelaZellen (3). Ob die Unterschiede zwischen verschiedenen
Zellarten unter Voraussetzung eines einheitlichen Transportmechanismus durch verschiedene Eigenschaften eines Carriers oder anderer am Transport beteiligter Proteine $z u$ erklären sind, bedarf weiterer Untersuchungen.

$\mathrm{Da}$ wir keinen stoffwechselabhängigen aktiven Transportmechanismus für $\mathrm{Zn}$ fanden, $\mathrm{Zn}$ aber intrazellulär stark angereichert ist und $\mathrm{Zn}$ andererseits mit intrazellulären Liganden stabile Chelate' 'bildet, kann die $\mathrm{Zn}$ Anreicherung in der Zelle durch. Komplexbildung erklärt werden.

\section{Literatur}

1. Dorn, F. und Th. Günther, diese Z. 8, 618 (1970). - 2. Lowry, O. H., N. Rosebrough, A. L. Farr und R. J. Randale, J. biol. Chemistry 193, 265 (1951). - 3. Cox, R. P., Mol. Pharmacol. 4, 510 (1968). - 4. Broda, E., H. Desser und G. FindenegG,
Naturwissenschaften 41, 361 (1964). - 5. RAthore, V. S., S. H. Wittwer, W. H. Jyung, V. P. S. Bajaj und M. W. Adams, Physiol. Plant. 23, 908 (1970). - 6. Wepemeyer, G., Comp. Biochem. Physiol. 26, 271 (1968).
Prof. Dr. Th. Günther 1000 Berlin 33

Arnimallee 22 